\title{
A NATURAL ORTHOGONAL BASIS OF EIGENFUNCTIONS OF THE HECKE ALGEBRA ACTING ON CAYLEY GRAPHS
}

\author{
JINGHUA KUANG \\ (Communicated by Dennis A. Hejhal)
}

\begin{abstract}
This paper discusses the representation of the Hecke algebra of $\mathrm{GL}_{2}\left(\mathbb{F}_{q}\right)$ on a class of Cayley graphs and gives a natural construction of an orthogonal basis of simultaneous eigenfunctions whose eigenvalues are the SotoAndrade Sums.
\end{abstract}

\section{INTRODUCTION AND NOTATION}

In [Ev], R. Evans constructed an orthogonal basis of simultaneous eigenfunctions of adjacency operators of a class of Cayley graphs, known to be Ramanujan graphs. In this paper, we give a natural construction of an orthogonal basis of simultaneous eigenfunctions of such adjacency operators using the theory of representations of $\mathrm{GL}_{2}\left(\mathbb{F}_{q}\right)$. The eigenvalues given by our basis are also the Soto-Andrade sums (see [Ev] and [S-A]). Our construction is natural and conceptual, hence ready to be extended to situations for bigger groups such as $\mathrm{Sp}_{n}\left(\mathbb{F}_{q}\right)$ and to local fields. One will see that the $\mathrm{H}$-functions constructed by Evans arise naturally from the theory of representations of $\mathrm{GL}_{2}\left(\mathbb{F}_{q}\right)$ : they are $\Psi_{a} * \eta_{\pi}$ for cuspidal representations $\pi$.

We set up some notation in this section. We discuss the structure of a Hecke algebra of $\mathrm{GL}_{2}\left(\mathbb{F}_{q}\right)$ in the next section. In Section 3 , we construct the abovementioned basis. In Section 4, we view the adjacency operators as elements of the Hecke algebra and find their eigenvalues to be Soto-Andrade sums.

After this paper was finished, the author received several papers from Audrey Terras. She and the UCSD group have done extensive study on the finite analog of the Poincare upper half plane, which is very closely related with the Hecke algebra we are discussing here. For the works of the UCSD group, see [A-V] and references therein.

Throughout this paper, $F=\mathbb{F}_{q}$ is a finite field with $q$ elements ( $q$ is a power of odd prime); $\varepsilon$ is a fixed non-square element in $F ; \chi$ is a fixed multiplicative character of $F^{*}$ of order $q-1$; and $\omega$ is a fixed multiplicative character of the quadratic extension $F(\sqrt{\varepsilon})$ of order $q^{2}-1$. Denote $\chi^{(q-1) / 2}$ by $s$, i.e. $s(x)$ is the quadratic symbol of $x$ in $F$. Let $N(z)$ denote the norm of $z \in F(\sqrt{\varepsilon})$

Received by the editors June 8, 1994.

1991 Mathematics Subject Classification. Primary 11T24, 12E20, 20 C33.

Key words and phrases. Hecke algebra, eigenfunctions, Soto-Andrade sums. 
to $F$. Fix a non-trivial additive character $\psi$ of $\mathbb{F}_{q}$. Let $G=\mathrm{GL}_{2}\left(\mathbb{F}_{q}\right)$,

$$
K=\left\{\left(\begin{array}{cc}
a & b \varepsilon \\
b & a
\end{array}\right) \in G\right\} \quad \text { and } P=\left\{\left(\begin{array}{cc}
y & x \\
0 & 1
\end{array}\right) \mid y \neq 0, x \in F\right\} .
$$

We have $K \cap P=\{1\}$. We identify $G / K$ with $P$. Notice that $G$ acts on $F(\sqrt{\varepsilon})$ by linear transformation:

$$
g(z)=\frac{a z+b}{c z+d}, \quad \text { for } g=\left(\begin{array}{ll}
a & b \\
c & d
\end{array}\right) \in G, z \in F(\sqrt{\varepsilon}) .
$$

$K$ is the isotropy group of $\sqrt{\varepsilon}$. Define a pseudo-distance $\Delta$ on $G$ as follows: $\Delta\left(g_{1}, g_{2}\right)=\frac{N\left(z_{1}-z_{2}\right)}{b_{1} b_{2}}$, if $g_{1}(\sqrt{\varepsilon})=z_{1}=a_{1}+b_{1} \sqrt{\varepsilon}, g_{2}(\sqrt{\varepsilon})=z_{2}=a_{2}+b_{2} \sqrt{\varepsilon}$.

It is easy to see that $K g K=K g^{\prime} K$ if and only if $\Delta(g, 1)=\Delta\left(g^{\prime}, 1\right)$. For a finite set $X$, let $|X|$ be its cardinality. Finally, we define a function $\delta$ on $F$ such that $\delta(y)=1$ if $y=1 ; \delta(y)=0$ if $y \neq 1$.

\section{The Hecke algebra $H(G, K)$}

Define $H(G, K)$ to be the linear space of all complex functions $\varphi$ on $G$ such that $\varphi\left(k_{1} g k_{2}\right)=\varphi(g)$ for all $g \in G, k_{1}, k_{2} \in K$. It is well known that $H(G, K)$ is an algebra under the multiplication defined by convolution: $\left(\varphi_{1} * \varphi_{2}\right)(g)=\sum_{h \in G} \varphi_{1}(h) \varphi_{2}\left(h^{-1} g\right)$. See [Kr] for more properties of the Hecke algebra.

Proposition 2.1. $H(G, K)$ is a commutative $\mathbb{C}$-algebra.

Proof. It suffices to prove that $\varphi_{1} * \varphi_{2}=\varphi_{2} * \varphi_{1}$ for all $\varphi_{1}, \varphi_{2} \in H(G, K)$. For that purpose and later use as well, we note that $K t K=K t^{-1} K$ since $\Delta(t, 1)=\Delta\left(t^{-1}, 1\right)$. Therefore,

$$
\begin{aligned}
\varphi_{1} * \varphi_{2}(t) & =\varphi_{1} * \varphi_{2}\left(t^{-1}\right) \\
& =\sum_{h \in G} \varphi_{1}(h) \varphi_{2}\left(h^{-1} t^{-1}\right) \\
& =\sum_{h \in G} \varphi_{2}\left((t h)^{-1}\right) \varphi_{1}(h) \\
& =\sum_{h \in G} \varphi_{2}(t h) \varphi_{1}\left(h^{-1}\right) \\
& =\sum_{g \in G} \varphi_{2}(g) \varphi_{1}\left(g^{-1} t\right)=\varphi_{2} * \varphi_{1}(t) .
\end{aligned}
$$

Since a double coset $K g K$ is determined by $\Delta(g, 1)$, we denote the double coset $K g K$ by $D_{a}$ if $\Delta(g, 1)=a$. There are $q$ double cosets, each corresponding to an element $a$ in $F$. Let $\varphi_{a}$ be the characteristic function of $D_{a}$. $H(G, K)$ is spanned by $\left\{\varphi_{a} \mid a \in F\right\}$. Hence $H(G, K)$ is a $q$-dimensional $\mathbb{C}$-algebra. Since $\mathbb{C}$ is an algebraically closed field, $H(G, K)$ is isomorphic to $\mathbb{C}^{\oplus q}$ as a $\mathbb{C}$-algebra. Therefore, there are $q$ non-zero idempotent elements $\eta_{1}, \ldots, \eta_{q} \in H(G, K)$ such that $\eta_{i} * \eta_{j}=0$ if $i \neq j$ and $\eta_{i} * \eta_{i}=\eta_{i}$. Now we can explicitly construct these idempotents. 
An irreducible representation $\pi$ of $G$ is called spherical if $\operatorname{Res}_{K}^{G}(\pi)$ contains the identity representation of $K$. For every spherical irreducible representation $\pi$ of $G$, define

$$
\eta_{\pi}^{\prime}(g)=\frac{1}{|K|} \sum_{k \in K} \sigma_{\pi}(k g),
$$

where $\sigma_{\pi}$ is the trace function of $\pi$. It is easy to see that $\eta_{\pi}^{\prime} \in H(G, K)$ and $\eta_{\pi}^{\prime}(1) \neq 0$.

Lemma 2.2. $\eta_{\pi_{1}}^{\prime} * \eta_{\pi_{2}}^{\prime}=0$ if $\pi_{1} \neq \pi_{2} ; \eta_{\pi}^{\prime} * \eta_{\pi}^{\prime}=\frac{|G|}{\operatorname{dim}(\pi)} \eta_{\pi}^{\prime}$.

Proof. Let $\pi_{1}, \pi_{2}$ be two spherical representations of $G$. Let us denote by $\langle$,$\rangle both invariant inner products on the spaces of \pi_{1}$ and $\pi_{2}$. Let $\{\mathbf{u}\}$ and $\left\{\mathbf{v}\right.$ be orthonormal bases of the spaces of $\pi_{1}$ and $\pi_{2}$ respectively. (If $\pi_{1} \cong \pi_{2}$, we assume $\{\mathbf{u}\}$ is identified with $\{\mathbf{v}\}$.) From the elementary theory of group representation, we have

$$
\begin{aligned}
\eta_{\pi_{1}}^{\prime} * \eta_{\pi_{2}}^{\prime}(g) & =\sum_{h \in G} \eta_{\pi_{1}}^{\prime}(h) \eta_{\pi_{2}}^{\prime}\left(h^{-1} g\right) \\
& =\sum_{h \in G}|K|^{-1} \sum_{k_{1} \in K} \sigma_{\pi_{1}}\left(k_{1} h\right)|K|^{-1} \sum_{k_{2} \in K} \sigma_{\pi_{2}}\left(k_{2} h^{-1} g\right) \\
& =|K|^{-2} \sum_{k_{1}, k_{2} \in K} \sum_{h \in G} \sigma_{\pi_{1}}(h) \sigma_{\pi_{2}}\left(k_{2} h^{-1} k_{1} g\right) \\
& =|K|^{-2} \sum_{k_{1}, k_{2} \in K} \sum_{h \in G} \sigma_{\pi_{1}}(h) \sigma_{\pi_{2}}\left(h^{-1} k_{1} g k_{2}\right) \\
& =|K|^{-2} \sum_{k_{1}, k_{2} \in K} \sum_{\mathbf{u}} \sum_{\mathbf{v}} \sum_{h \in G}\left\langle\pi_{1}(h) \mathbf{u}, \mathbf{u}\right\rangle\left\langle\pi_{2}\left(h^{-1}\right) \pi_{2}\left(k_{1} g k_{2}\right) \mathbf{v}, \mathbf{v}\right\rangle \\
& =0 \quad \text { if } \pi_{1} \not \pi_{2} .
\end{aligned}
$$

But if $\pi_{1} \cong \pi_{2} \cong \pi$, we have

$$
\begin{aligned}
\eta_{\pi_{1}}^{\prime} * \eta_{\pi_{2}}^{\prime}(g) & =|K|^{-2} \sum_{k_{1}, k_{2} \in K} \sum_{\mathbf{u}} \sum_{\mathbf{v}} \frac{|G|}{\operatorname{dim}(\pi)}\langle\mathbf{u}, \mathbf{v}\rangle\left\langle\pi\left(k_{1} g k_{2}\right) \mathbf{v}, \mathbf{u}\right\rangle \\
& =|K|^{-2} \sum_{k_{1}, k_{2} \in K} \sum_{\mathbf{u}} \frac{|G|}{\operatorname{dim}(\pi)}\left\langle\pi\left(k_{1} g k_{2}\right) \mathbf{u}, \mathbf{u}\right\rangle \\
& =|K|^{-2} \sum_{k_{1}, k_{2} \in K} \frac{|G|}{\operatorname{dim}(\pi)} \sigma_{\pi}\left(k_{1} g k_{2}\right) \\
& =\frac{|G|}{\operatorname{dim}(\pi)}|K|^{-1} \sum_{k \in K} \sigma_{\pi}(k g)=\frac{|G|}{\operatorname{dim}(\pi)} \eta_{\pi}^{\prime}(g) .
\end{aligned}
$$

From the table on page 70 of [P-S], there are $q$ spherical irreducible representations of $G$, and they are

$\rho_{\nu_{j}}\left(\nu_{j}=\omega^{j(q-1)}, j=1, \ldots,(q-1) / 2\right)$ with dimension $=q-1 ;$

$\mathbf{1}_{G}$ with dimension $=1$;

$\rho(s, s)$ with dimension $=q$;

$\rho\left(\chi^{j}, \bar{\chi}^{j}\right)(j=1, \ldots,(q-3) / 2)$ with dimension $=q+1$. 
We note here that $\operatorname{dim} \operatorname{Hom}\left(\mathbf{1}_{K}, \operatorname{Res}_{K}^{G} \pi\right)=1$, hence $\eta_{\pi}^{\prime}(1)=1$ for these $\pi$. For these spherical irreducible representations $\pi$, define $\eta_{\pi}=\frac{\operatorname{dim}(\pi)}{|G|} \eta_{\pi}^{\prime}$. Denote $\eta_{0}=\eta_{1} ; \eta_{j}=\eta_{\rho\left(\chi^{j}, \bar{\chi}^{j}\right)}, j=1, \ldots,(q-3) / 2 ; \eta_{(q-1) / 2}=\eta_{\rho(s, s)}$ and $\eta_{(q-1) / 2+j}=\eta_{\rho_{\nu_{j}}}, j=1, \ldots,(q-1) / 2$. Then we have

Theorem 2.3. $\eta_{j}, j=0, \ldots, q-1$, are the $q$ idempotents in $H(G, K)$ such that $\eta_{i} * \eta_{j}=0$ if $i \neq j$ and $\eta_{j} * \eta_{j}=\eta_{j}$.

Lemma 2.4. $\bar{\eta}_{j}(h)=\eta_{j}\left(h^{-1}\right)=\eta_{j}(h)$, i.e. these $\eta_{j}$ are real-valued functions.

Proof. The reason is that all the spherical representations used to construct $\eta_{j}$ are isomorphic to their contragradients and $K h K=K h^{-1} K$.

\section{THE REPRESENTATIONS OF $H(G, K)$}

Let $L^{2}(P)$ be the space of all (square-summable) complex functions on $P$. An element of $L^{2}(P)$ can also be viewed as a right $K$-invariant function on $G$. $H(G, K)$ acts on $L^{2}(P)$ by convolution, i.e. for $\varphi \in H(G, K), f \in L^{2}(P)$,

$$
T_{\varphi}(f)(p)=(f * \varphi)(p)=\sum_{h \in G} f\left(p h^{-1}\right) \varphi(h)=|K| \sum_{p_{1} \in P} f\left(p p_{1}^{-1}\right) \varphi\left(p_{1}\right) .
$$

Define an inner product $\langle$,$\rangle on L^{2}(P)$ by setting $\left\langle f_{1}, f_{2}\right\rangle=\sum_{p \in P} f_{1}(p) \bar{f}_{2}(p)$. It is easy to see $\left\langle T_{\varphi}\left(f_{1}\right), f_{2}\right\rangle=\left\langle f_{1}, T_{\hat{\varphi}}\left(f_{2}\right)\right\rangle$, where $\hat{\varphi}(h)=\bar{\varphi}\left(h^{-1}\right)$. Hence the commutative finite-dimensional $\mathbb{C}$-algebra $H(G, K)$ acts selfadjointly on $L^{2}(P)$. There exists a basis of simultaneous eigenfunctions of $H(G, K)$. Now we are going to construct such a basis explicitly.

We recall that $\chi$ is a fixed multiplicative character of $F^{*}$ of order $q-1$, $\psi$ is a fixed non-trivial additive character of $F$ and $\delta(y)=1$ if $y=1$; $\delta(y)=0$ if $y \neq 1$. Let us now define some right $K$-invariant functions on $G$, or equivalently functions on $P$. For each character $\chi^{i}$ of $F^{*}$, define $\chi_{i}(g)=$ $\chi^{i}(y)$ where $g K=\left(\begin{array}{ll}y & x \\ 0 & 1\end{array}\right) K$. We note that $\chi_{i}\left(p_{1} p_{2}\right)=\chi_{i}\left(p_{1}\right) \chi_{i}\left(p_{2}\right)$ for $p_{1}, p_{2} \in$ $P$. For each $a \in F^{*}$, define $\Psi_{a}(g)=\delta(y) \psi(a x)$ where $g K=\left(\begin{array}{ll}y & x \\ 0 & 1\end{array}\right) K$. We need the following lemma.

Lemma 3.1. Let $\mathbf{u}_{a}$ be a vector in the space of a non-trivial spherical representation $\pi$ of $G$ such that $\pi\left(\left(\begin{array}{ll}1 & u \\ 0 & 1\end{array}\right)\right) \mathbf{u}_{a}=\psi(a u) \mathbf{u}_{a}$ for all $u \in F$. Then $\sum_{k \in K}\left\langle\pi(k) \mathbf{u}_{a}, \mathbf{u}_{a}\right\rangle \neq 0$.

Proof. Since $\operatorname{Res}_{K}^{G} \pi$ contains $\mathbf{1}_{K}$, let $V_{0}$ be the $K$-invariant subspace on which $K$ acts according to $\mathbf{1}_{K}$. It suffices to show that $\mathbf{u}_{a}$ is not perpendicular to $V_{0}$ under the $G$-invariant inner product $\langle$,$\rangle . Assume the contrary, if$ $\left\langle\mathbf{u}_{a}, V_{0}\right\rangle=0$, then $\left\langle\pi(k) \mathbf{u}_{a}, V_{0}\right\rangle=\left\langle\mathbf{u}_{a}, \pi\left(k^{-1}\right) V_{0}\right\rangle=0$ and $V_{0}^{\perp}$ contains a $K$-and- $\left(\begin{array}{ll}1 & u \\ 0 & 1\end{array}\right)$-invariant subspace of $\pi$. But $G$ is generated by $K$ and all the elements $\left(\begin{array}{ll}1 & u \\ 0 & 1\end{array}\right)$, hence $V_{0}^{\perp}$ contains a $G$-invariant subspace of $\pi$, a contradiction to the irreducibility of $\pi$.

Now we can prove

Theorem 3.2. $\left\{\chi_{i}, T_{\eta_{i}}\left(\Psi_{a}\right)=\Psi_{a} * \eta_{i}, i=1, \ldots, q-1, a \in F^{*}\right\}$ is an orthogonal basis consisting of simultaneous eigenfunctions of $H(G, K)$. Moreover, 
$T_{\eta_{j}}\left(\chi_{i}\right)=|K|\left\langle\eta_{j}, \chi_{i}\right\rangle \chi_{i}$, and $T_{\eta_{j}}\left(\Psi_{a} * \eta_{i}\right)=\Psi_{a} * \eta_{i}$ if $i=j ; \quad T_{\eta_{j}}\left(\Psi_{a} * \eta_{i}\right)=0$ if $i \neq j$.

Proof. First of all, we have

$$
\left\langle\chi_{i}, \chi_{j}\right\rangle=\sum_{p \in P} \chi_{i}(p) \bar{\chi}_{j}(p)=\sum_{x \in F} \sum_{y \in F^{*}} \chi^{i}(y) \bar{\chi}^{j}(y)= \begin{cases}0 & \text { if } i \neq j \\ q(q-1) & \text { if } i=j\end{cases}
$$

Recall from Lemma 2.4 that $\eta_{j}(h)=\eta_{j}\left(h^{-1}\right)=\bar{\eta}_{j}(h)$. If $i \neq j$, we have

$$
\left\langle T_{\eta_{i}}\left(\Psi_{a}\right), T_{\eta_{j}}\left(\Psi_{b}\right)\right\rangle=\left\langle\Psi_{a}, T_{\eta_{j} * \hat{\eta}_{i}}\left(\Psi_{b}\right)\right\rangle=\left\langle\Psi_{a}, T_{\eta_{j} * \eta_{i}}\left(\Psi_{b}\right)\right\rangle=0
$$

If $i=j$,

$$
\begin{aligned}
& \left\langle T_{\eta_{i}}\left(\Psi_{a}\right), T_{\eta_{i}}\left(\Psi_{b}\right)\right\rangle=\left\langle\Psi_{a}, T_{\eta_{i}}\left(\Psi_{b}\right)\right\rangle \\
& \quad=\sum_{p \in P} \Psi_{a}(p) \sum_{h \in G} \bar{\Psi}_{b}\left(p h^{-1}\right) \bar{\eta}_{i}(h) \\
& =|K| \sum_{p_{1} \in P} \bar{\eta}_{i}\left(p_{1}\right) \sum_{p \in P} \Psi_{a}(p) \bar{\Psi}_{b}\left(p p_{1}^{-1}\right) \\
& =|K| \sum_{u \in F} \sum_{v \in F^{*}} \bar{\eta}_{i}\left(\left(\begin{array}{ll}
v & u \\
0 & 1
\end{array}\right)^{-1}\right) \sum_{y \in F^{*}} \sum_{x \in F} \Psi_{a}\left(\left(\begin{array}{cc}
y & x \\
0 & 1
\end{array}\right)\right) \bar{\Psi}_{b}\left(\left(\begin{array}{cc}
v y & u y+x \\
0 & 1
\end{array}\right)\right) \\
& =|K| \sum_{u \in F} \sum_{v \in F^{*}} \eta_{i}\left(\left(\begin{array}{ll}
v & u \\
0 & 1
\end{array}\right)\right) \sum_{y \in F^{*}} \sum_{x \in F} \delta(y) \psi(a x) \delta(v y) \bar{\psi}(b u y+b x) \\
& =|K| \sum_{u \in F} \eta_{i}\left(\left(\begin{array}{ll}
1 & u \\
0 & 1
\end{array}\right)\right) \bar{\psi}(b u) \sum_{x \in F} \psi((a-b) x)=0 \quad \text { if } a \neq b .
\end{aligned}
$$

If $\left.a=b,\left\langle T_{\eta_{i}}\left(\Psi_{a}\right), T_{\eta_{i}}\left(\Psi_{b}\right)\right\rangle=|K| q \sum_{u \in F} \eta_{i}\left(\begin{array}{ll}1 & u \\ 0 & 1\end{array}\right)\right) \bar{\psi}(a u)$. Let $\pi$ be the representation of $G$ that gives $\eta_{i}$ as in Section 2. Let us denote $U=\left\{\left(\begin{array}{ll}1 & u \\ 0 & 1\end{array}\right) \mid u \in F\right\}$ and $\mathbb{C u}=$ the $\mathbb{C}$-span of $\mathbf{u}$. If $\operatorname{dim}(\pi)>1$, from Theorem 16.1 of $[\mathrm{P}-\mathrm{S}]$ and Frobenius Reciprocity, we have the decomposition

$$
\left.\pi\right|_{U}=\bigoplus_{c \in F^{*}} \mathbb{C u} \oplus W_{0}
$$

Here and in the sequel, for $c \in F^{*}, \pi\left(\left(\begin{array}{ll}1 & u \\ 0 & 1\end{array}\right)\right) \mathbf{u}_{c}=\psi(c u) \mathbf{u}_{c}$ for all $u \in F$, and $\left.\pi\right|_{U}$ acts trivially on $W_{0}$. The dimension of $W_{0}=0,1$, or 2 depending on whether the dimension of $\pi=q-1, q$, or $q+1$ respectively. Let $\left\{\mathbf{u}_{c} \mid c \in F\right\}^{\prime}$ be an orthonormal basis of the space of $\pi$ such that $\pi\left(\left(\begin{array}{ll}1 & u \\ 0 & 1\end{array}\right)\right) \mathbf{u}_{c}=\psi(c u) \mathbf{u}_{c}$. Here and in the sequel, the prime' indicates that for $c=0, \mathbf{u}_{0}$ does not appear, or stands for one basis element from $W_{0}$, or stands for two basis elements from 
$W_{0}$ depending on the dimension of $W_{0}=0$, or 1 , or 2 . Then

$$
\begin{aligned}
\eta_{i}\left(\left(\begin{array}{cc}
1 & u \\
0 & 1
\end{array}\right)\right) & =\frac{1}{|K|} \sum_{k \in K} \sigma_{\pi}\left(k\left(\begin{array}{ll}
1 & u \\
0 & 1
\end{array}\right)\right) \\
& =\frac{1}{|K|} \sum_{k \in K} \sum_{\mathbf{u}_{c}}\left\langle\pi\left(k\left(\begin{array}{cc}
1 & u \\
0 & 1
\end{array}\right)\right) \mathbf{u}_{c}, \mathbf{u}_{c}\right\rangle \\
& =\frac{1}{|K|} \sum_{k \in K} \sum_{c \in F}^{\prime}\left\langle\pi(k) \psi(c u) \mathbf{u}_{c}, \mathbf{u}_{c}\right\rangle \\
& =\frac{1}{|K|} \sum_{k \in K} \sum_{c \in F}^{\prime} \psi(c u)\left\langle\pi(k) \mathbf{u}_{c}, \mathbf{u}_{c}\right\rangle
\end{aligned}
$$

and

$$
\begin{aligned}
\left\langle T_{\eta_{i}}\left(\Psi_{a}\right), T_{\eta_{i}}\left(\Psi_{a}\right)\right\rangle & =|K| q \sum_{u \in F} \eta_{i}\left(\left(\begin{array}{cc}
1 & u \\
0 & 1
\end{array}\right)\right) \bar{\psi}(a u) \\
& =q \sum_{u \in F} \sum_{k \in K} \sum_{c \in F}^{\prime} \psi(c u)\left\langle\pi(k) \mathbf{u}_{c}, \mathbf{u}_{c}\right\rangle \bar{\psi}(a u) \\
& =q \sum_{k \in K} \sum_{c \in F}^{\prime}\left\langle\pi(k) \mathbf{u}_{c}, \mathbf{u}_{c}\right\rangle \sum_{u \in F} \psi((c-a) u) \\
& =q^{2} \sum_{k \in K}\left\langle\pi(k) \mathbf{u}_{a}, \mathbf{u}_{a}\right\rangle \neq 0,
\end{aligned}
$$

from Lemma 3.1. To verify $\left\langle\chi_{i}, T_{\eta_{j}}\left(\Psi_{a}\right)\right\rangle=0$, we calculate

$$
\begin{aligned}
T_{\eta_{j}}\left(\chi_{i}\right)(p) & =\chi_{i} * \eta_{j}(p)=\sum_{h \in G} \chi_{i}\left(p h^{-1}\right) \eta_{j}(h) \\
& =|K| \sum_{p_{1} \in P} \chi_{i}\left(p p_{1}^{-1}\right) \eta_{j}\left(p_{1}\right)=|K|\left\langle\eta_{j}, \chi_{i}\right\rangle \cdot \chi_{i}(p) .
\end{aligned}
$$

Now

$$
\begin{aligned}
\left\langle\chi_{i}, T_{\eta_{j}}\left(\Psi_{a}\right)\right\rangle & =\left\langle T_{\hat{\eta}_{j}}\left(\chi_{i}\right), \Psi_{a}\right\rangle=\left\langle T_{\eta_{j}}\left(\chi_{i}\right), \Psi_{a}\right\rangle \\
& =\left\langle\eta_{j}, \chi_{i}\right\rangle\left\langle\chi_{i}, \Psi_{a}\right\rangle=\left\langle\eta_{j}, \chi_{i}\right\rangle \sum_{p \in P} \chi_{i}(p) \bar{\Psi}_{a}(p) \\
& =\left\langle\eta_{j}, \chi_{i}\right\rangle \sum_{x \in F} \sum_{y \in F^{*}} \chi_{i}(y) \delta(y) \bar{\psi}(a x)=0 .
\end{aligned}
$$

\section{EIGENVALUES OF THE ADJACENCY OPERATORS}

Recall that all the $\varphi_{a}$, the characteristic functions of the double cosets $D_{a}$ ( $a \in F$ ), also make up a basis of $H(G, K)$. Each $T_{\varphi_{a}}$ acts on $L^{2}(P)$ in the same way up to a factor of $|K|$ as the adjacency operator $A_{a}$ acts on the Cayley graphs in [Ev]. We are interested in the eigenvalues of $A_{a}$, or equivalently those of $T_{\varphi_{a}}$ in the canonical representation of $H(G, K)$ on $L^{2}(P)$. Each $\eta_{i}$ takes the same value on every element in a double coset $D_{a}$. We denote the common value by $\eta_{i}\left(D_{a}\right)$. Let $S_{a}=D_{a} \cap P$. We have the following 
Theorem 4.1. The eigenvalues of $A_{a}$ on $\chi_{i}$ are $|K| \sum_{p \in S_{a}} \chi_{i}(p) \quad(i=1, \ldots, q-$ 1). The eigenvalues of $A_{a}$ on $\Psi_{b} * \eta_{i}$ are $\frac{|G|}{\operatorname{dim} \pi_{i}}\left|S_{a}\right||K| \eta_{i}\left(D_{a}\right)(i=1, \ldots, q-1$, $\forall b)$, where $\pi_{i}$ is the representation that gives $\eta_{i}$ as in Section 2.

Proof. The first statement is from direct calculation:

$$
\begin{aligned}
A_{a}\left(\chi_{i}\right)(p) & =\chi_{i} * \varphi_{a}(p)=\sum_{h \in G} \chi_{i}\left(p h^{-1}\right) \varphi_{a}(h) \\
& =|K| \sum_{p_{1} \in P} \chi_{i}\left(p p_{1}\right) \varphi_{a}\left(p_{1}^{-1}\right)=|K| \sum_{p_{1} \in S_{a}} \chi_{i}\left(p p_{1}\right) \\
& =\left(|K| \sum_{p_{1} \in S_{a}} \chi_{i}\left(p_{1}\right)\right) \cdot \chi_{i}(p) .
\end{aligned}
$$

For the second statement, we note at first that

$$
\begin{aligned}
\left\langle\eta_{i}, \eta_{i}\right\rangle & =\sum_{p \in P} \eta_{i}(p) \bar{\eta}_{i}(p)=\frac{1}{|K|} \sum_{h \in G} \eta_{i}(h) \eta_{i}\left(h^{-1}\right) \\
& =\frac{1}{|K|} \eta_{i} * \eta_{i}(1)=\frac{\eta_{i}(1)}{|K|}=\frac{\operatorname{dim} \pi_{i}}{|K||G|} .
\end{aligned}
$$

Since $A_{a}\left(\Psi_{b} * \eta_{i}\right)=\Psi_{b} * \eta_{i} * \varphi_{a}$ and $\eta_{i} * \varphi_{a}=B_{i}(a) \eta_{i}$ for some number $B_{i}(a)$, the eigenvalue is equal to the number $B_{i}(a)$. Now

$$
\begin{aligned}
\left\langle\eta_{i} * \varphi_{a}, \eta_{i}\right\rangle & =\sum_{p \in P} \eta_{i} * \varphi_{a}(p) \bar{\eta}_{i}(p) \\
& =\sum_{p \in P} \sum_{h \in G} \eta_{i}\left(p h^{-1}\right) \varphi_{a}(h) \bar{\eta}_{i}(p) \\
& =|K| \sum_{p \in P} \sum_{p_{1} \in P} \eta_{i}\left(p p_{1}^{-1}\right) \varphi_{a}\left(p_{1}\right) \bar{\eta}_{i}(p) \\
& =|K| \sum_{p \in P} \sum_{p_{1} \in S_{a}} \eta_{i}\left(p p_{1}^{-1}\right) \eta_{i}(p) \\
& =\sum_{p_{1} \in S_{a}} \sum_{h \in G} \eta_{i}(h) \eta_{i}\left(h^{-1} p_{1}^{-1}\right)=\sum_{p_{1} \in S_{a}} \eta_{i} * \eta_{i}\left(p_{1}^{-1}\right) \\
& =\sum_{p_{1} \in S_{a}} \eta_{i}\left(p_{1}^{-1}\right)=\left|S_{a}\right| \eta_{i}\left(D_{a}\right)
\end{aligned}
$$

Hence

$$
B_{i}(a)=\frac{\left\langle\eta_{i} * \varphi_{a}, \eta_{i}\right\rangle}{\left\langle\eta_{i}, \eta_{i}\right\rangle}=\frac{|G|}{\operatorname{dim} \pi_{i}}|K|\left|S_{a}\right| \eta_{i}\left(D_{a}\right)
$$

From the discussion in [Ev], one knows that $\eta_{i}\left(D_{a}\right)$ and $\sum_{p \in S_{a}} \chi_{i}(p)$ are the Soto-Andrade sums. It is of great interest to evaluate or even to estimate the functions $\eta_{i}\left(D_{a}\right)$, which are essentially the eigenvalues of the Hecke algebra. The Soto-Andrade sums have been estimated at least in two ways: see [Ka] and [Li] respectively. See [A-V] and references therein for more information on the eigenvalues. 


\section{REFERENCES}

[Ev] R. Evans, Character sums as orthogonal eigenfunctions of adjacency operators for Cayley graphs, Finite Fields: Theory, Applications, and Algorithms, Contemp. Math., vol. 168, Amer. Math. Soc., Providence, RI, 1994.

[Ka] N. Katz, Estimates for Soto-Andrade sums, J. Reine Angew. Math. 438 (1993), 143-161.

[Kr] A. Krieg, Hecke algebras, Mem. Amer. Math. Soc., No. 435, Amer. Math. Soc., Providence, RI, 1990.

[Li] W. Li, A survey of Ramanujan graphs, Proc. Conf. on Arithmetic Geometry and Coding Theory at Luminy, 1993.

[S-A] J. Soto-Andrade, Geometrical Gel' fand models, tensor quotients and Weil representations, The Arcata Conference on Representations of Finite Groups (Arcata, CA, 1986), Proc. Sympos. Pure Math., vol. 47, Part 2, Amer. Math. Soc., Providence, RI, 1987, pp. 305-316.

[P-S] I. Piatetski-Shapiro, Complex representation of $\mathrm{GL}_{2}\left(\mathbb{F}_{q}\right)$, Contemp. Math., vol. 16, Amer. Math. Soc., Providence, RI, 1983.

[A-V] J. Angel, N. Celniker, S. Poulos, A. Terras, C. Trimble, and E. Velasquez, Special functions on finite upper half planes, Contemp. Math., vol. 138, Amer. Math. Soc., Providence, RI, 1992.

Department of Mathematics, PennState-Fayette, Uniontown, Pennsylvania 15401

E-mail address: jkuang@fert1.fe.psu.edu 